\title{
SHIP DETECTION WITH COSMO-SKYMED PINGPONG DATA USING THE DUAL-POL RATIO ANOMALY DETECTOR
}

\author{
Armando Marino * \\ The Open University, \\ Engineering and Innovation, \\ Milton Keynes, United Kingdom
}

\begin{abstract}
Extensive work has been carried out on detecting ships using space-borne Synthetic Aperture Radar (SAR) systems. However, the identification of small vessels is still challenging especially when the sea conditions are rough. In this work, a new detector is proposed based on dual-polarized incoherent SAR images. Small ships have a stronger cross polarization accompanied by a higher cross- over co-polarization ratio compared to sea. This is the rational at the base of the detector.

The new detector is tested with dual-polarization $\mathrm{HH} / \mathrm{HV}$ PINGPONG Cosmo-SkyMed images acquired over the North Sea. The test area is near Rotterdam where a large number of ships are expected.
\end{abstract}

Index Terms - SAR, polarimetry, ship detection, COSMOSkyMed

\section{SAR POLARIMETRY}

Based on its polarization characteristics, a single target can be characterized using the scattering (Sinclair) matrix or equivalently a scattering vector $\underline{k}$ [1]. In the lexicographic basis system and in case of reciprocity and monostatic system, the scattering vector is $\underline{k}_{L}=[H H, \sqrt{2} H V, V V]$, where $H$ stands for linear horizontal and $V$ for linear vertical (therefore the $H V$ image is obtained transmitting a linear vertical polarization and receiving the linear horizontal one). $H H$ and $V V$ are often referred to as co-channels and $H V$ or $V H$ are the cross-channels.

* The data were provided by the project CORSAIR27. COSMO-SkyMed Product - (C)ASI 2016 processed under license from ASI - Agenzia Spaziale Italiana. All rights reserved. Distributed by e-GEOS. The collaboration in this work was supported by the ESA/NRCSS Dragon-4 program under the project 32235

$\dagger$

\author{
Pasquale Iervolino ${ }^{\dagger}$ \\ University of Surrey, \\ Surrey Space Centre \\ , Guildford, United Kingdom
}

\section{DUAL-POLARIZATION RATIO ANOMALY DETECTOR (DPOLRAD)}

\subsection{Dimensionless detector}

An algorithm is proposed for the detection of small vessels in high sea state. The Dual-Polarization Ratio Anomaly Detector (DPolRAD) was already successfully used with Sentinel1 data to detect icebergs embedded in sea ice [2]. This work presents the very first test of the DPolRAD for ship detection. Additionally, we will use here COSMO-SkyMed data, which acquires at a different frequency band than Sentinel-1. The algorithm is based on the observation that ships exhibit a different polarimetric behaviour compared to the sea surface [3]. Specifically, the cross polarization channel and the ratio between cross- and co-polarizations (here referred to as depolarization ratio) increases. Ships are composed by a large collection of structures (mostly metallic) $[4,5]$. The orientation of these structures is generally random and therefore they are expected to have a large cross-polarisation contribution [6]. Additionally, the presence of multiple reflections (specifically even-bounces) between the sea and the ship hull can increase the depolarization ratio if the ship is not perfectly oriented along an azimuth line.

Two boxcar filters are applied over the HV and HH intensity images, exploiting two different window sizes: a smaller window or test window $w_{\text {test }}$ and a larger window or training window $w_{\text {train }}$. The detector can be written as:

$$
\Lambda=\frac{\left\langle|H V|^{2}\right\rangle_{\text {test }}-\left\langle|H V|^{2}\right\rangle_{\text {train }}}{\left\langle|H H|^{2}\right\rangle_{\text {train }}}>T_{\Lambda} .
$$

where \langle\rangle$_{\text {test }}$ and \langle\rangle$_{\text {train }}$ identify the spatial average using the test and training windows respectively and $T_{\Lambda}$ is a threshold.

After some mathematical manipulations it is possible to gain some physical understanding of the proposed formula. If the averages are expressed explicitly the following equation can be derived:

$$
\Lambda=\rho_{\text {ring }} \frac{1+c}{R \rho^{-1}+c R H V^{-1}}-\rho_{\text {train }}
$$


$\rho$ stands for cross-over-co polarization ratio, in the following defined as depolarization ratio. As mentioned previously, this observable is sensitive to the presence of orientated structures. $R \rho$ is the ratio between the $\rho$ inside the test area over the one in the ring around the test area (i.e. $R \rho=\frac{\rho_{\text {test }}}{\rho_{\text {ring }}}$ ). $R H V$ is the ratio of the $\mathrm{HV}$ intensity in the test area over the ring area (i.e. $R H V=\frac{\left\langle|H V|^{2}\right\rangle_{\text {test }}}{\left\langle|H V|^{2}\right\rangle_{\text {ring }}}$ ). $c$ is a factor such that $N_{\text {train }}=c N_{\text {test }}$ where $N_{\text {train }}$ and $N_{\text {test }}$ are the number of pixels inside the training and test windows. $\rho_{\text {ring }}$ and $\rho_{\text {train }}$ are the depolarization ratios in the ring and the entire training windows respectively.

Analyzing some special conditions, it is possible to gain insights into the nature of the detector:

(1) It is easy to proof that $\Lambda$ is equal to zero if the depolarization ratio and the $\mathrm{HV}$ intensity do not change between the ring and the test area. This is because $\rho_{\text {ring }}=\rho_{\text {train }}$ and $R \rho=R H V=1$. As a consequence, homogeneous areas will provide a $\Lambda$ that is equal to zero.

(2) If and only if the depolarization ratio and the HV intensity increase significantly between the ring and test area, then $\Lambda$ becomes very large. An easy way to test this is by considering the limit of $R \rho$ and $R H V$ going to infinity:

$$
\lim _{\substack{R \rho \rightarrow \infty, R H V \rightarrow \infty}} \Lambda=\rho_{\text {ring }} \frac{1+c}{0+c 0}-\rho_{\text {tot }}=\infty
$$

Clearly, $R \rho$ and $R H V$ will never reach infinity in real data due to the noise level (i.e. the values in the ring areas cannot be exactly zero).

(3) Finally, if the volume or multiple reflections decrease drastically from the ring to the test area (e.g. a pool of open water in multi-year sea ice), then $\Lambda$ becomes negative. A way to see this is by analyzing the limit of $\Lambda$ when $R \rho$ and $R H V$ go to zero.

$$
\lim _{\substack{R \rho \rightarrow 0, R H V \rightarrow 0}} \Lambda=\rho_{\text {ring }} \frac{1+c}{\infty+c \infty}-\rho_{\text {tot }}=-\rho_{\text {tot }}
$$

To summarize, if a ship enters the test window, the value of $\Lambda$ increases triggering a detection. Additionally, please note that this detector does not need the phase information of each polarimetric channels but only their magnitude. It is therefore appropriate for systems that do not acquire the polarisation channels coherently.

\subsection{Contrast enhancement: HV-DPolRAD}

$\Lambda$ is large when there is an increase in volume or oriented scattering, equals to zero on homogeneous targets and is negative if there is a decrease in oriented scattering. Such detector is built as a ratio between intensities and therefore it is scale invariant. This is a very valuable property for a polarimetric observable, however scale invariance may be disadvantageous for some detection task. For instance, if the signal is very low and close to the noise floor, the detector may become noisy. An easy way out is by multiplying the detector by an intensity or magnitude image. In this context, the cross polarization channel should be preferred because it shows a higher contrast between ship and clutter:

$$
I=\Lambda \cdot\left\langle|H V|^{2}\right\rangle
$$

In this version, if a pixel of the HV intensity image presents an anomaly in volume or oriented reflections, then it is multiplied by a large number. If it presents a homogeneous area, then it is multiplied by zero and if it presents a decrease in volume or oriented reflections, then it becomes negative. This enhances the contrast between anomalies in volume/orientedreflections and clutter.

\section{TEST WITH COSMO-SKYMED PINGPONG DATA}

\subsection{Presentation of data}

In this section, the algorithm is tested using dual-polarized HH/HV PINGPONG Cosmo SkyMed images. The resolution is $15 \mathrm{~m}$. 10 images were acquired under the CORSAIR call of opportunity. The location of the data is in the North Sea, near the harbour of Rotterdam. This is one of the busiest harbours in the North Sea allowing us to capture several vessels. The acquisitions were done in the months of October and November 2016, which should help observing rough sea conditions. For the sake of brevity only two acquisitions will be presented in this abstract, but in the final paper the analysis will be extended to the full dataset.

\subsection{Visual inspection}

In this preliminary analysis, only two dates from the $8^{\text {th }}$ and $16^{\text {th }}$ of November are analysed. The wind speed inside the harbour was showing gusts of $35 \mathrm{Km} / \mathrm{h}$ and $48 \mathrm{Km} / \mathrm{h}$ respectively.

Figure 1 presents the magnitude of $\mathrm{HH}$ and $\mathrm{HV}$ for the $8^{\text {th }}$ of November. They represent an area outside the harbour of Rotterdam with two lines of docked ships. There is no AIS information for this image, but the ships are located in an area that is dedicated to docking.

The capability of the iDPolRAD to enhance the contrast between ships and sea is tested here. The test window considers $7 \times 7$ pixels, while the training window is $55 \times 55$ pixels. The results for the iDPolRAD is shown in Figure 1 at the bottom left. The scaling used for this image is exactly the same exploited for the HV magnitudes. The image appear darker, because the sea clutter is strongly reduced. In this images, when the iDPolRAD is negative (i.e. reduction of volume 

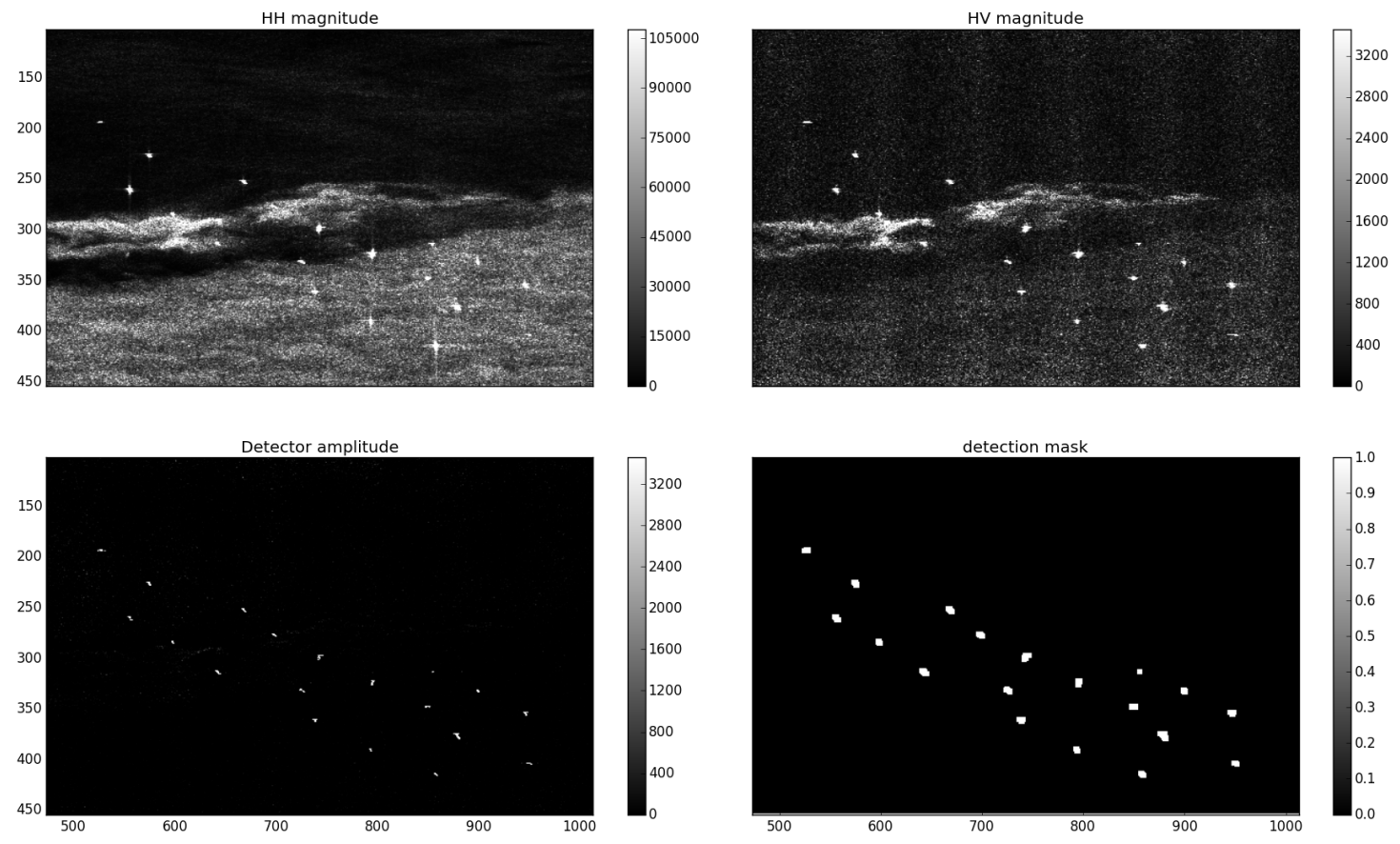

Fig. 1. Detection results with iDPolRAD using COSMO SkyMed PINGPONG HH/HV images (North Sea near Rotterdam, 8/11/2016). (Top left) HH magnitude; (Top right) HV magnitude; (Bottom left) IDPolRAD, usin gthe same scaling than HV; (Bottom right) Detection mask using an CA-CFAR with parameter 6. Boxcar filter: $7 \times 7$ pixels. COSMO-SkyMed Product (C)

or oriented reflections) the iDPolRAD is set to zero. On the other hand, bright isolated points remain bright.

The same analysis is repeated for the data on the $16^{\text {th }}$ of November and presented in Figure 2

\subsection{Detection masks}

The detection masks obtained with the iDPolRAD are shown in Figure 1 and Figure 2 at the bottom right. The algorithm used to set the threshold is a Cell-Averaging Constant False Alarm Rate (CA-CFAR) (Figure 1 bottom right). The algorithm is based on the finding that the distribution of the sea clutter (for the positive values of iDPolRAD) can be approximated with an exponential distribution. This is the base for the selection of a CA-CFAR. The factor we used for the CACFAR is equal to 6 , since this is a value largely adopted for ship detection [6]. Please note, when calculating the mean of the clutter it is important to only exploit the positive values. For the pure sake of visualisation the detected targets in the detection mask were enlarged using a Dilate filter with a $5 \times 5$ pixels kernel. This is the only reason why the detected targets look bigger than the ships.

The proposed algorithm is able to detect the ships even in areas where the sea background is particularly strong.

\section{REFERENCES}

[1] S. R. Cloude, Polarisation: Applications in Remote Sensing, Oxford University Press, Oxford, UK, 2009.

[2] A. Marino, W Dierking, and C. Wesche, "A depolarization ratio anomaly detector to identify icebergs in sea ice using dual-polarization SAR images," vol. 54, no. 9, pp. 5602-5615, 2016.

[3] A. Marino, "A notch filter for ship detection with polarimetric SAR data," IEEE Journal of Selected Topics in Applied Earth Observations and Remote Sensing, vol. 6, no. 3, pp. 1219 - 1232, June 2013.

[4] F. Nunziata, M. Migliaccio, and C.E. Brown, "Reflection symmetry for polarimetric observation of man-made metallic targets at sea," IEEE Journal of Oceanic Engineering, vol. 37, no. 3, pp. 384-394, July 2012.

[5] P. Iervolino, R. Guida, and P. Whittaker, "A new GLRTbased ship detection technique in SAR images," IEEE IGARSS, Milan, Itay, 2015.

[6] D. J. Crisp, "The State-of-the-Art in ship detection in Synthetic Aperture Radar imagery," Australian Government Department of Defence, 2004. 

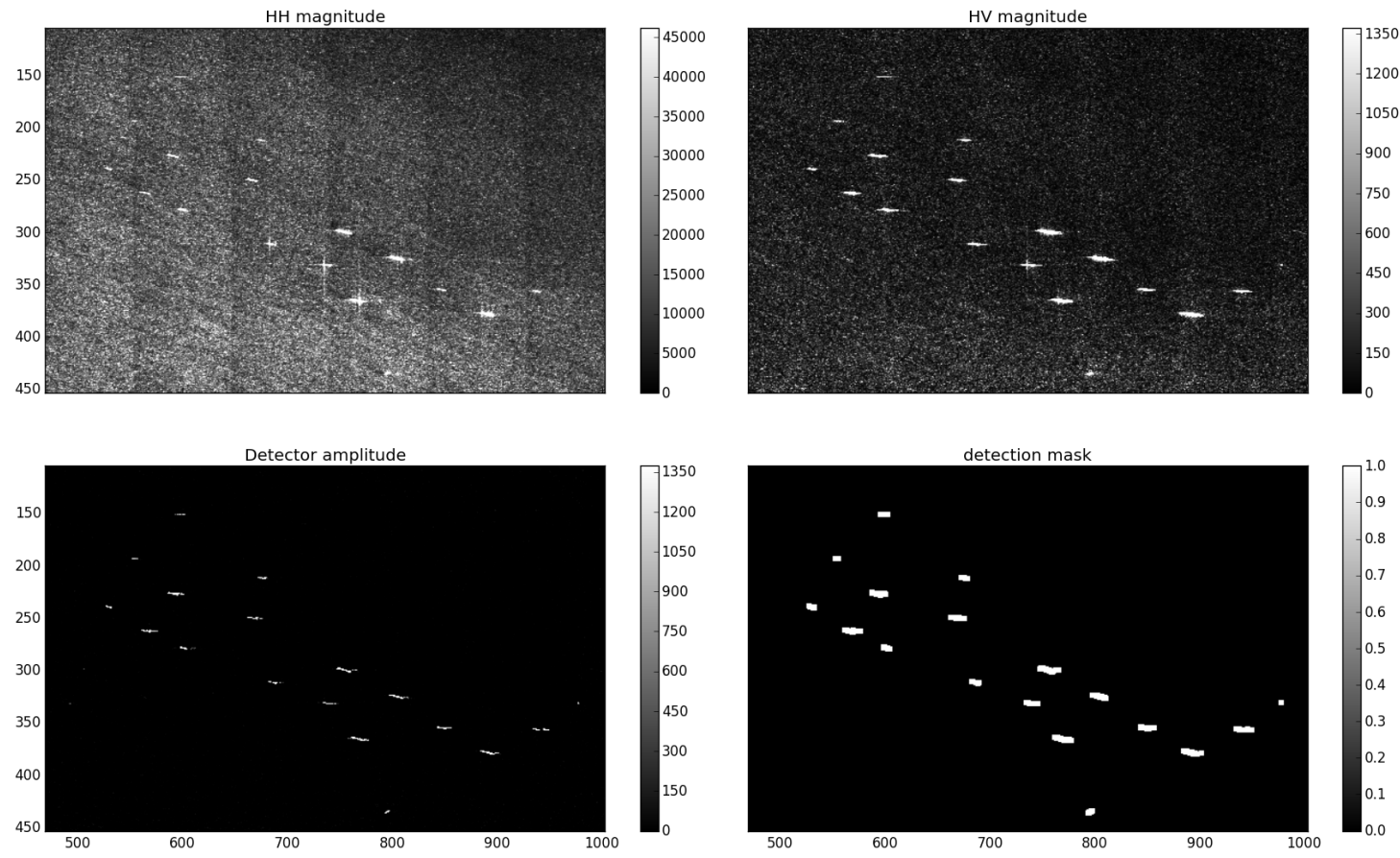

Fig. 2. Detection results with iDPolRAD using COSMO SkyMed PINGPONG HH/HV images (North Sea near Rotterdam, 16/11/2016). (Top left) HH magnitude; (Top right) HV magnitude; (Bottom left) IDPolRAD, using the same scaling than HV; (Bottom right) Detection mask using an CA-CFAR with parameter 6. Boxcar filter: $7 \times 7$ pixels. COSMO-SkyMed Product (C) 